\title{
Nonadiabatic simulation to study the photoexcited phase change in $\mathrm{Ge}_{2} \mathrm{Sb}_{2} \mathrm{Te}_{5}$
}

\author{
Shinnosuke Hattori*, Fuyuki Shimojo \\ Department of Physics, Kumamoto University, Kumamoto 860-8555, Japan \\ *Corresponding author: 134d9001@kumamoto-u.ac.jp
}

\begin{abstract}
Nonadiabatic quantum molecular dynamics (NAQMD) simulations are performed to understand the fast photoexcited phase change of $\mathrm{Ge}_{2} \mathrm{Sb}_{2} \mathrm{Te}_{5}$ (GST), which is widely used for data storage. NAQMD approach, which simulates energy transfer from excited electrons to the finite temperature ionic system, shows that GST is fast heated up in few picoseconds toward the melting point. This result is considered to be one of the realistic phase change processes through the liquid phase, and also provides an insight on the non-thermal phase change, which is a promising property to improve the speed of phase change.
\end{abstract}

Key words: GeSbTe, phase-change material, ab initio molecular dynamics, nonadiabatic quantum simulation

\section{INTRODUCTION}

Photoexcited phase change phenomena are extremely important for data extensive consumer products like rewritable optical disks and solid-state memories. Further improvements are demanded to realize low power and higher throughput storage devices by atomistic level design of candidate materials. However, the details of fast energy transfer processes from excited electrons are not well understood due to the experimental difficulties. Recently, femtosecond laser pulse and time resolved optical pump-probe techniques directly observed surprisingly short time period atomistic vibrations including coherent phonons [1]. Also, theoretical approaches using the framework of density functional theory (DFT) provided remarkable insights from the reproduction of phase change processes by ab initio molecular dynamics (MD) simulations [2]. Static DFT calculations are also a powerful tool to evaluate the amorphous local structure and to predict phase change models [3]. However, most of the simulations do not involve the electronic excitation process. Xian-Bin Li et al. evaluated the effect of electronic excitations by removing valence band electrons [4]. This simulation found that the excitation makes lowers the critical amorphization temperature. It is, however, unclear what is the contribution of heating up due to the electronic excitations.

In such a situation, nonadiabatic quantum MD (NAQMD) simulation [5] is one of the valuable methodologies incorporating nonadiabatic electronic transitions to study photoexcitation dynamics of electrons and ions. So far, NAQMD simulations have been performed for charge separation phenomena in dendrimer [6] and organic solar cell materials [7].

Here, we firstly performed NAQMD simulations for a typical phase change material $\mathrm{Ge}_{2} \mathrm{Sb}_{2} \mathrm{Te}_{5}$ (GST) to investigate the time evolution of both the excited electrons and ions.

\section{SIMULATION METHODOLOGY}

NAQMD simulations are based on Tully's fewest switches surface-hopping approach to describe the nonadiabatic electron-ion dynamics with transition probabilities calculated by time-dependent DFT (TDDFT) [8]. In this study, transition probabilities of multiple excited electrons were estimated by the time dependent Kohn Sham (TD-KS) scheme [6]. In addition, excited state atomic forces were effectively and accurately evaluated by a non-self consisted approximation [5]. Projector augmented wave (PAW) method was employed to calculate electronic states. Energy cut off was $200 \mathrm{eV}$ and MD time step was 2 fs. We use 194 atoms $(42 \mathrm{Ge}+44 \mathrm{Sb}+108 \mathrm{Te})$ in a cubic super cell of side $18.5 \AA$. Periodic boundary conditions are applied to all Cartesian directions.

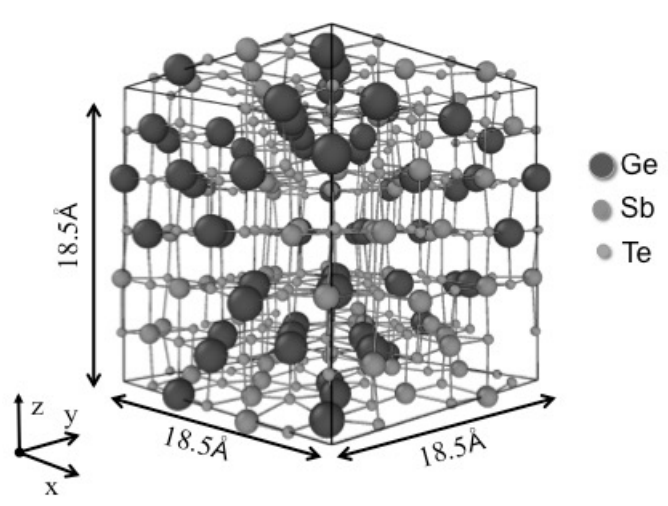

Fig. 1. Initial configuration for $\mathrm{MD}$ simulations of $\mathrm{Ge}_{2} \mathrm{Sb}_{2} \mathrm{Te}_{5}$. The large, middle and small spheres represent $\mathrm{Ge}, \mathrm{Sb}$ and $\mathrm{Te}$ atoms, respectively. 
In a previous study, DFT calculations showed that the $\mathrm{Ge}_{2} \mathrm{Sb}_{2} \mathrm{Te}_{5}$ optical band gap depends on the atomic configuration, i.e. $\mathrm{Sb}$ and vacancy positions [9]. In this study, Ge atoms are randomly exchanged for $\mathrm{Sb}$ atoms in the rock salt structure of GeTe [10], and vacancies are introduced at $\mathrm{Ge}$ sites for the charge neutrality to make an initial configuration. We also observed that the band gap is dependent on $\mathrm{Sb}$ and vacancy positions. Therefore, initial configuration was selected so that the optical band gap is close to experimental optical band gap as much as possible (The band gap obtained by this work is $0.38 \mathrm{eV}$, while the experiment value is approximately $0.5 \mathrm{eV}[10])$.

To relax the atomic structure at the finite temperature condition, we performed a standard $a b$ initio MD simulation for $2 \mathrm{ps}$ in the canonical ensemble at $300 \mathrm{~K}$ without electronic excitation. The calculated pressure was confirmed to be almost $0 \mathrm{~Pa}$. NAQMD simulations were performed in 20 ps (10000 step) in the microcanonical ensemble to simulate the local adiabatic heating up due to the energy transfer from the electronic to the atomic degrees of freedom. The optimal number of excited electrons in GST has been evaluated by the optical absorption efficiency [4]. It was estimated that a typical femtosecond laser setup could readily generate excited electrons in excess of $9 \%$. In this study, 1, 2, 3 and $4 \%$ electrons were excited at an initial step from the top area of valence band to the bottom area of conduction band to study the effects of the number of excited electrons on the structure.

\section{RESULTS AND DISCUSSIONS}

The time evolution of the atomic configuration obtained by the NAQMD simulations for the cases of 1-4 \% electronic excitations is shown in Fig. 2, where the snapshots at $0,5,10,15$, and $20 \mathrm{ps}$ are shown. The initial configuration $(0 \mathrm{ps})$ is identical for all simulations. It is seen that, at 1 and $2 \%$ excitations, the rock salt structure is primarily retained, except that a few atoms have unexpectedly large displacements from their equilibrium positions. On the other hand, at 3 and $4 \%$ excitations, a disordered phase is obtained.

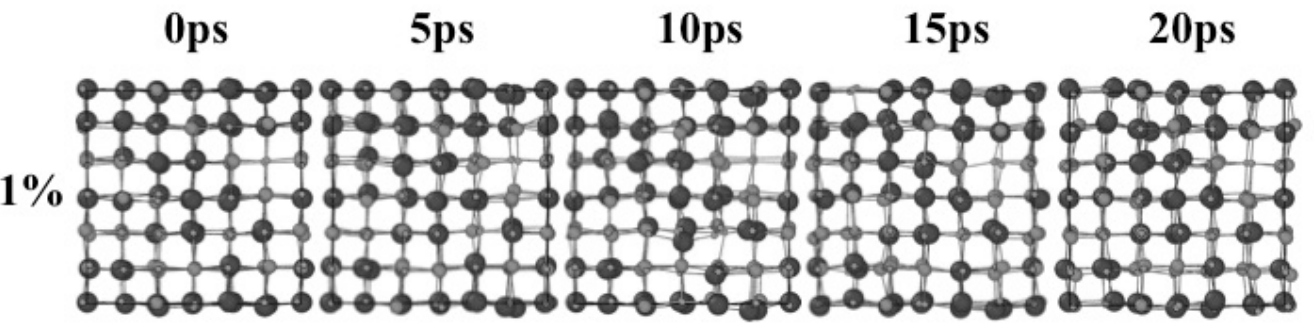

$2 \%$
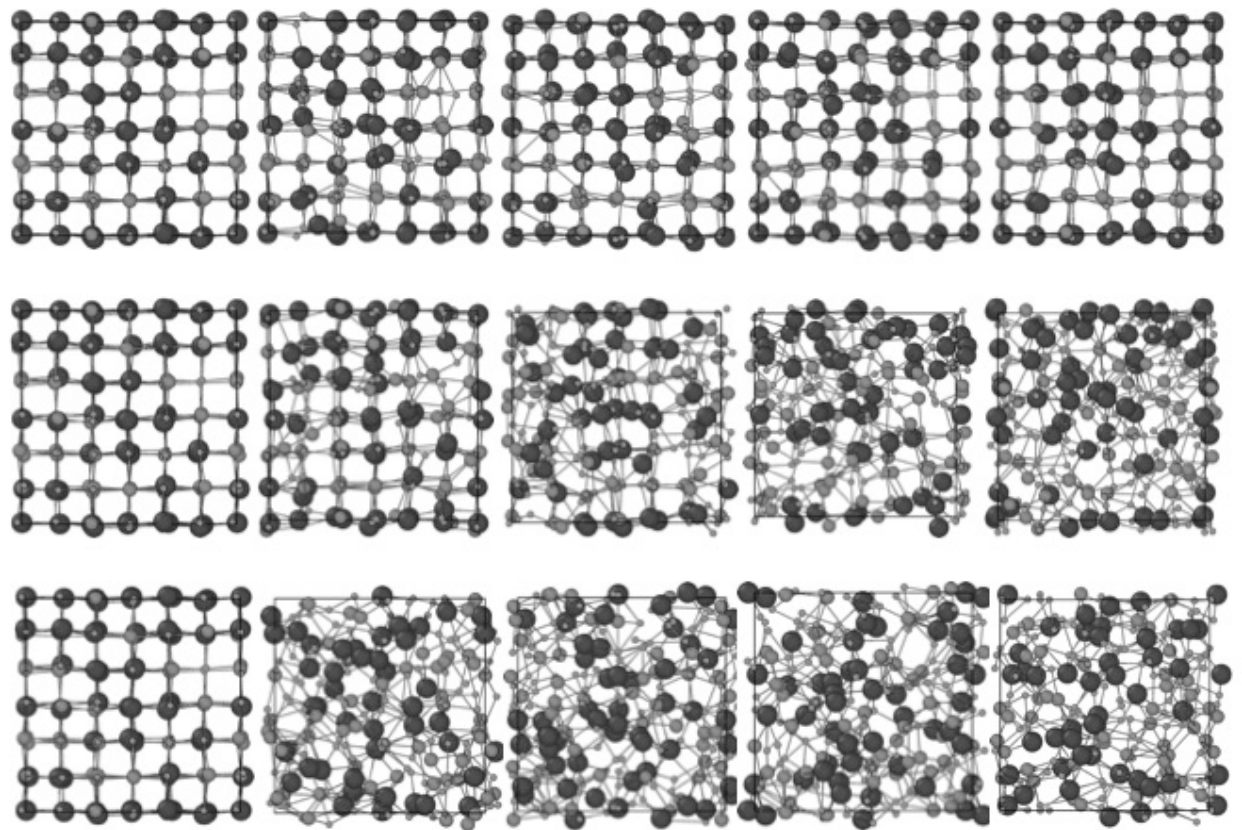

Fig. 2. Snapshots of the atomic configuration obtained by the NAQMD simulations for $\mathrm{Ge}_{2} \mathrm{Sb}_{2} \mathrm{Te}_{5}$ with the different number of excited electron 1-4\%. The large, middle and small spheres represent $\mathrm{Ge}, \mathrm{Sb}$ and $\mathrm{Te}$ atoms, respectively. 

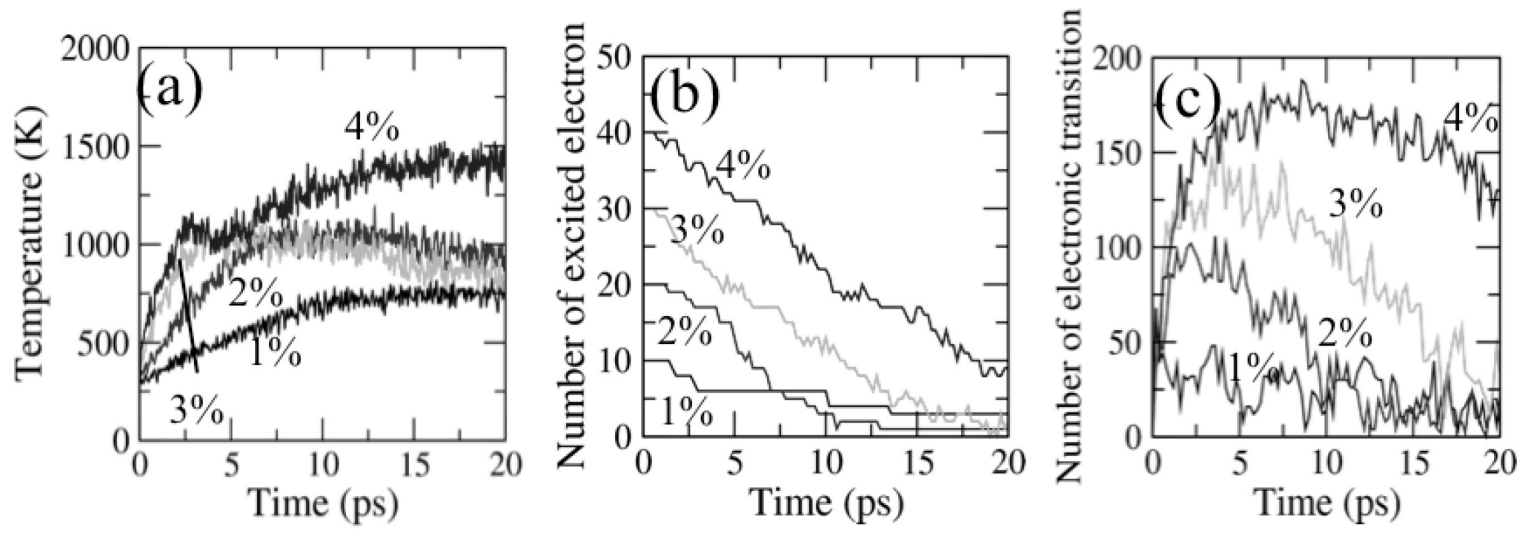

Fig. 3. Time evolution of (a) temperature of the ion system, (b) the number of excited electrons and (c) the number of electronic transitions for $20 \mathrm{ps}$.

Figures 3(a), 3(b), and 3(c) show the time evolution of temperature of ions, the number of excited electrons and the number of electronic transitions, respectively. The temperature of ions has been estimated by taking the average of kinetic energy at each time step. The number of excited electron has been obtained by counting the number of electrons in the conduction bands. In all simulations, the temperature is increased and the heating speed depends on the number of excited electrons. As shown in Fig. 2, the dynamic structural change to a disordered phase is observed in the simulations for the 3 and $4 \%$ excitations, while such structural change is not observed in the cases of 1 and $2 \%$ excitations. It is, therefore, concluded that the amorphization boundary exists between the 2 and $3 \%$ excitations. Nevertheless, the temperature profile for the 2 and $3 \%$ excitations are similar to each other (Fig. 3(a)), both of which are increased to around the melting point, $1000 \mathrm{~K}$ (The experimental melting point in GST is $900 \mathrm{~K}$ [4]). The difference between the 2 and $3 \%$ excitations can be seen in the time evolution of the number of excited electrons, i.e. the excited electrons in the case of $2 \%$ excitations are more rapidly decreased at $5 \mathrm{ps}$ at which the temperature approaches the melting point, as shown in Fig. 3(b). On the other hand, the decreasing rate of the number of the excited electrons is rather constant during $20 \mathrm{ps}$ at the $3 \%$ excitations. When $4 \%$ excitations are excited, the decreasing rate is also almost constant as shown in Fig. 3(b). Interestingly, the decreasing rate of excited electrons for the $1 \%$ excitations is the slowest among the cases of 1-4\% excitations, which is caused by the fact that the transition probability depends on the temperature through the Boltzmann factor [8]. It is also shown that the number of electronic transitions shown in Fig. 3 (c) is highly corresponding to the temperature profile (Fig. 3 (a)). When the number of electronic transitions increases, the temperature of the system also increases in all cases. However, after the number of electronic transitions turns to decrease, the temperature keeps increasing. In the case of $4 \%$ excitations, the temperature is still increasing at $20 \mathrm{ps}$, while the number of electronic transitions decreases gradually from approximately $10 \mathrm{ps}$. In other cases, the temperature is almost constant or decreases slightly over 10 ps because the number of electronic transitions decreases largely. In the cases of 1 and $2 \%$ excitations, the number of excited electron is not changed over $15 \mathrm{ps}$, while the values of the number of electronic transitions fluctuate. This means that the remaining excited electrons are still transferred between the conduction bands, and that the system is in an excited state, i.e. the system does not return to the ground state within $20 \mathrm{ps}$. It seems that the life time of the last few electrons is rather long as shown in Fig. 3(b). From the snapshots for the $3 \%$ excitations displayed in Fig. 2, we see that the rock salt structure remains until $5 \mathrm{ps,}$, and a highly disordered state appears around $10 \mathrm{ps}$. This result indicates that the phase changing process needs enough number of excited electrons as well as a locally high enough temperature.

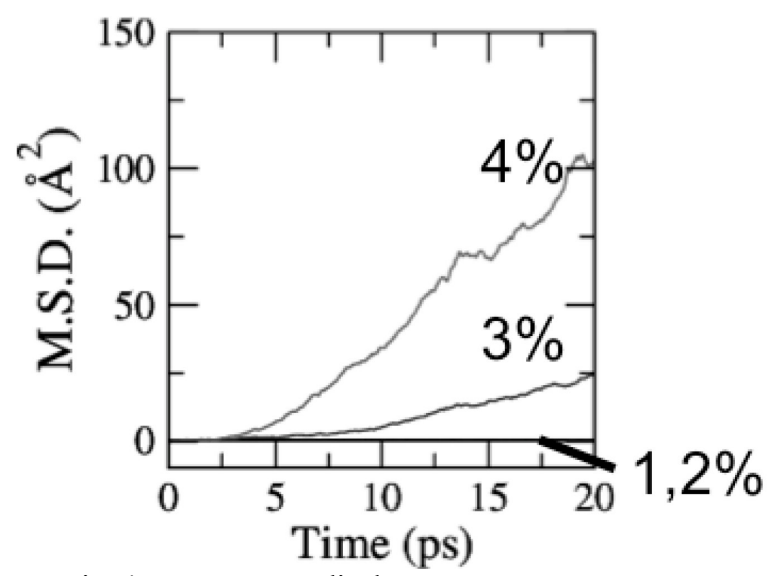

Fig. 4. Mean square displacements.

Figure 4 shows the mean square displacements (M. S. D.) averaged over all atoms. We see that a liquid phase appears when 3 and $4 \%$ electrons are excited because the M. S. D. increases linearly. The M.S.D. for the $4 \%$ excitations is consistent with that obtained for the liquid state at $1100 \mathrm{~K}$ reported in ref. [4].

The final solid structure after melting state will be crystalline or amorphous, strongly depending on the cooling rates [11]. When the liquid states were suddenly 
quenched to $300 \mathrm{~K}$ at $20 \mathrm{ps}$, they would become an amorphous phase.

Figure 5 shows the time evolution of the total number of wrong bonds i.e. $\mathrm{Ge}-\mathrm{Ge}, \mathrm{Ge}-\mathrm{Sb}$ and $\mathrm{Sb}-\mathrm{Sb}$ bonds, which do not exist in octahedral $(\mathrm{Ge}, \mathrm{Sb})-\mathrm{Te}_{6}$ units in the rock salt structure. Two atoms are considered to form a bond when the distance between them is shorter than 3.3 $\AA$. In the $3 \%$ excitations case, these bonds were generated continuously from $2.5 \mathrm{ps}$ at which the system still keeps the solid phase. Similar bond change has been also seen in the non-thermal phase change by the prior photoexcited simulation effect using the removal of $9 \%$ of the valence band electrons at $600 \sim 700 \mathrm{~K}[4,12]$. The NAQMD simulations with non-adiabatic effects have demonstrated that the critical number of excited electrons for the occurrence of the phase change is just $3 \%$, much smaller than $9 \%$. Therefore, the nonadiabatic effects are important to consider the amorphization processes.

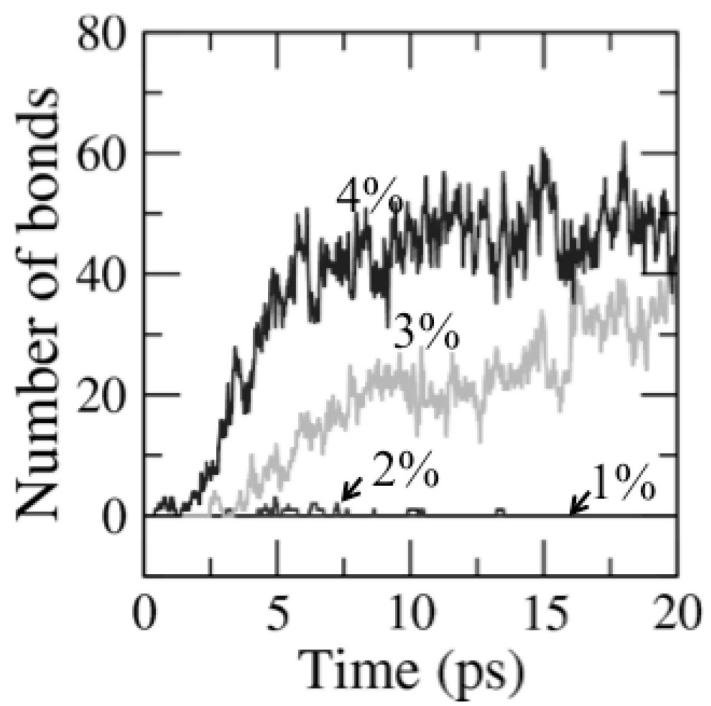

Fig. 5. Time evolution of the total number of $\mathrm{Ge}-\mathrm{Ge}, \mathrm{Ge}-\mathrm{Sb}$ and $\mathrm{Sb}-\mathrm{Sb}$ bonds.

\section{SUMMARY}

NAQMD simulations incorporating the electronic excitation have been performed to study the structural change processes in $\mathrm{Ge}_{2} \mathrm{Sb}_{2} \mathrm{Te}_{5}$. Our simulations suggest that the phase-change occurs due to the thermal effects in addition to the influence of the anti-bonding character of the excited electronic states.

\section{ACKNOWLEDGEMENTS}

The authors acknowledge useful discussions with Dr. Paulo S. Branicio. Computations were performed using the computer facilities at the Research Institute for Information Technology, Kyushu University, and the Supercomputer Center, Institute for Solid State Physics, The University of Tokyo.

\section{REFERENCES}

[1] Paul Fons et al., Phys. Rev. B 90, 094305 (2014).

[2] J. Akola and R. O. Jones, J. Phys.: Condens. Matter

20, 465103 (2008)

[3] Shinya Hosokawa et al., J. Appl. Phys. 111, 083517

(2012)

[4] Xian-Bin Li et al., Phys. Rev. Lett. 107, 015501

(2011)

[5] Fuyuki Shimojo et al., Comput. Phys. Commun., 184 1-8 (2013).

[6] Satoshi Ohmura et al., Appl. Phys. Lett. 98, 113302

(2011)

[7] Weiwei Mou et al., Appl. Phys. Lett. 100, 113302

(2012)

[8] J.C. Tully, J. Chem. Phys. 93, 1061 (1990)

[9] Caravati et al., J. Phys.: Condens. Matter 22, 399801 (2010)

[10] N. Yamada et al., J. Appl. Phys. 69, 2849 (1991)

[11] Paulo S. Branicio et al, MRS Online Proceeding Library 1431, f03-05 (2012)

[12] A.V. Kolobov et al. Phys. Status Solidi B 251, 7, 1297-1308 (2014)

(Received February 1, 2015; Accepted May 29, 2015) 